\title{
Synthesis and incorporation of a furan-modified adenosine building block for DNA interstrand crosslinking $\dagger$
}

\author{
Anup M. Jawalekar, ${ }^{a}$ Marieke Op de Beeck, ${ }^{b}$ Floris L. van Delft ${ }^{* a}$ and Annemieke Madder ${ }^{b}$ \\ Received 28th October 2010, Accepted 17th January 2011 \\ DOI: $10.1039 / \mathrm{c0cc04667a}$
}

$2^{\prime}$ - $O$-(3-(Furan-2-yl)propyl)adenosine was synthesized and evaluated for interstrand crosslink (ICL) formation in DNA duplexes. In situ oxidation of the furan moiety with NIS showed rapid crosslink formation to $\mathrm{dA}$ and $\mathrm{dC}$, while $\mathrm{dT}$ and $\mathrm{dG}$ were inactive.

Oligonucleotides that form interstrand crosslinks (ICLs) have found widespread applications in chemical biology research areas. Therefore, chemical and enzymatic methods have been developed to incorporate crosslinks into helical regions of DNA and RNA. ${ }^{1}$ Examples include the synthesis of a duplex incorporating a preformed crosslinked dinucleotide $e^{2,3}$ or postsynthetic modification of duplexes by bifunctional crosslinking reagents. ${ }^{4,5}$ However, in some cases, the site-specific introduction of a crosslink is problematic due to formation of a mixture of monoadducts, intrastrand and interstrand crosslinks. Introduction of reactive moieties at a specific position within the duplex can circumvent the selectivity issue as is shown for photocrosslinking with 4 -thiouridine, ${ }^{6}$ 5-bromouridine, ${ }^{7}$ 5-methyleneaminouridine ${ }^{8}$ or 8 -azidoadenosine. ${ }^{9}$ Furthermore, a thio-modified oligonucleotide (ON) can form disulfide bonds post-synthetically. ${ }^{10,11}$ However such ICLs are highly dependent on the proper positioning of thiol groups and, more importantly, require modification of both strands. More recent studies on site-specific ICL formation involve modified phenylselenyl derivatives of thymidine or 5-methyl-2'-deoxycytidine, ${ }^{12}$ 1,4-dioxobutane abasic lesion, ${ }^{13}$ alkyl-connected 2-amino6-vinylpurine ${ }^{14}$ and 4-amino-6-oxo-2-vinylpyrimidine. ${ }^{15} \mathrm{We}$ have earlier developed a complementary methodology incorporating furan-modified 2'-amido-uridine and an acyclic building block to give a site-specific ICL upon oxidation with $\mathrm{N}$ bromosuccinimide (NBS). ${ }^{16,17}$

\footnotetext{
$\overline{{ }^{a} \text { Institute for Molecules and Materials, Radboud University Nijmegen, }}$ Heyendaalseweg 135, NL-6525 AJ, Nijmegen, the Netherlands. E-mail: F.vanDelft@science.ru.nl; Fax: + 3124 3653393; Tel: + 31243652373

${ }^{b}$ Laboratory for Organic and Biomimetic Chemistry, Department of Organic Chemistry, Krijgslaan 281, S4, 9000, Gent, Belgium.E-mail: annemieke.madder@Ugent.be; Fax: + 329 2644498; Tel: + 3292644472

$\dagger$ Electronic supplementary information (ESI) available: Synthesis and characterization of compounds 2-7 and oligonucleotides, RP-HPLC profiles, PAGE of cross-linked oligonucleotides. See DOI: 10.1039/ c0cc04667a
}

First crosslinking results obtained for the furan-modified $2^{\prime}$-amido-uridine with complementary adenine (A) were further extended into a more detailed selectivity study against all canonicals using a more synthetically accessible acyclic furanmodified building block. In the latter case, strong selectivity for crosslinking to its opposite canonical A or cytidine (C) was observed, without formation of crosslinks to neighboring or distant bases. ${ }^{17}$

In this context, furan-modification on other canonicals than uridine and the potential impact on the crosslinking process have not been evaluated yet. From this point of view, we became interested in evaluating the scope of furan-modified purines for ICL. We here wish to report on the synthesis and incorporation of $2^{\prime}$-O-(3-(furan-2-yl)propyl)adenosine as a building block for crosslinking of DNA duplexes.

From a synthetic point of view, $2^{\prime}$-modification was an obvious choice as nucleobase modification will in most cases be unfavourable for hybridization. Taking into account that $2,2^{\prime}$-anhydrointermediates are only feasible for pyrimidines, a $2^{\prime}$-amido modification of adenosine is difficult to achieve. Therefore, we decided to take advantage of our previously developed $2^{\prime}$-O-alkylation strategy of adenosine. ${ }^{18}$ Moreover, linkage of a furan moiety via an ether instead of an amide connection has the additional advantage of more conformational flexibility.

Following our earlier protocol, without requiring protection, the adenosine $2^{\prime}-\mathrm{OH}$ was selectively propargylated (Scheme 1) to give nucleoside 2 in $45 \%$ yield. Next, the exocyclic amine was benzoylated $(\mathbf{2} \rightarrow \mathbf{3})$, followed by microwave-assisted Sonogashira coupling with 2-iodofuran ${ }^{19}$ to afford compound 4 . Upon subsequent hydrogenation, the desired 3-(furan-2-yl)propyl derivative $\mathbf{5}$ was obtained in excellent yield. Further conversion into the corresponding $5^{\prime}-O$-DMT-3'-O-phosphoramidite 7 was carried out using standard methodology and proceeded without incidence. ${ }^{18}$

Having the phosphoramidite 7 at hand, four ON sequences were synthesized (Fig. 1) with the furanyl compound in between two identical canonicals. Sequences 8-11 were obtained uneventfully in high purity (99\%) after Sep-pak cartridge purification, which indicated the smooth incorporation of phosphoramidite 7 .

Subsequently, each individual sequence 8-11 was subjected to hybridization experiments with a total of four complementary sequences, by varying only the base opposite to our modified 


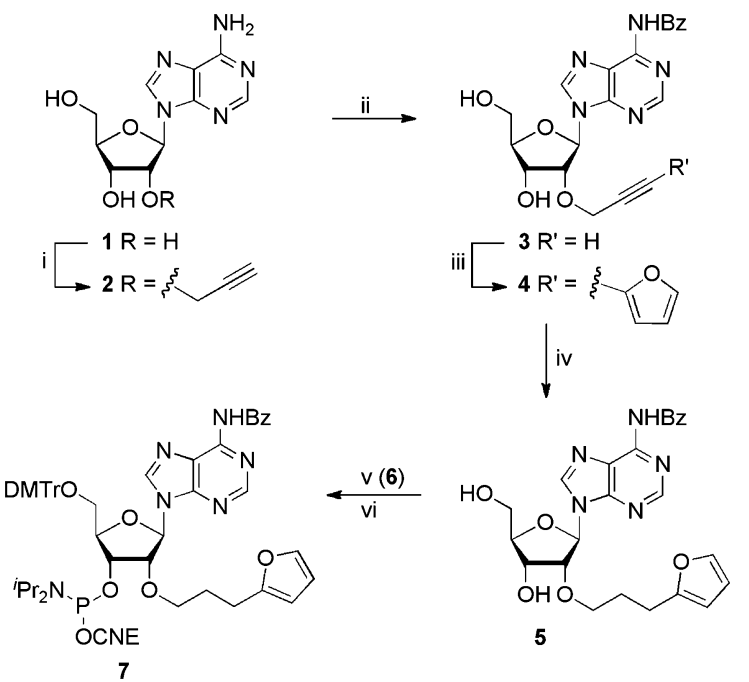

Scheme 1 Reagents and conditions: (i) $\mathrm{NaH}, \mathrm{TBAI}, \mathrm{CH} \equiv \mathrm{CCH}_{2} \mathrm{Br}$, DMF, $55^{\circ} \mathrm{C}, 2 \mathrm{~d}(45 \%)$; (ii) $\mathrm{TMSCl}, \mathrm{BzCl}, \mathrm{C}_{5} \mathrm{H}_{5} \mathrm{~N}, 0{ }^{\circ} \mathrm{C}, 6 \mathrm{~h}(92 \%)$; (iii) 2-iodofuran, $\mathrm{Pd}\left(\mathrm{PPh}_{3}\right)_{2} \mathrm{Cl}_{2}, \mathrm{CuI}, \mathrm{Et}_{3} \mathrm{~N} / \mathrm{DMF}$, $\mu$ wave, $55^{\circ} \mathrm{C}, 30 \mathrm{~min}$ (95\%); (iv) $\mathrm{H}_{2} / \mathrm{Pd}-\mathrm{C}, 20$ bar, $30{ }^{\circ} \mathrm{C}, 15 \min (93 \%)$; (v) $\mathrm{DMTrCl}$, $\mathrm{C}_{5} \mathrm{H}_{5} \mathrm{~N}$, rt, 16 h (77\%); (vi) $\mathrm{NC}\left(\mathrm{CH}_{2}\right)_{2} \mathrm{OP}\left(\mathrm{NiPr}_{2}\right) \mathrm{Cl}$, DIPEA, $\mathrm{CH}_{2} \mathrm{Cl}_{2}$, rt, 3 h $(92 \%)$.

\section{5'-d(CTGACGCA*CTGC) 3' (8) \\ 5'-d(CTGACGTA*TTGC) 3' (9) \\ 5'-d(CTGACGAA*ATGC)3' (10) \\ 5'-d(CTGACGGA*GTGC)3' (11)}

Fig. 1 Synthetic oligonucleotides with $\mathrm{A}^{*}$ as the modified adenosine.

adenosine $A^{*}$. It was interesting to find that incorporation of $A^{*}$ led to destabilization $\left(1.6-9.6{ }^{\circ} \mathrm{C}\right)$ in most cases, with exception of a stabilizing effect, i.e. $1.7{ }^{\circ} \mathrm{C}$ or $5.1{ }^{\circ} \mathrm{C}$, for the hybrids of sequence $\mathbf{1 1}$ with the complementary sequence having $\mathrm{A}$ or $\mathrm{C}$ opposite to $\mathrm{A}^{*}$, respectively (Table $\mathrm{S} 1$, ESI $\dagger$ ). The increase in duplex stability can possibly be attributed to the stacking interactions between the neighbouring nucleotides ${ }^{20}$ and the flexible $2^{\prime}$-O-alkyl substitution so as to accommodate well in the major groove.

Next, the potential of the furan-modified building block for crosslinking was evaluated. Crosslinking reactions were performed at $25^{\circ} \mathrm{C}$ at $0.02 \mathrm{mM}$ concentration in $10 \mathrm{mM}$ phosphate buffer at $\mathrm{pH} 7$, containing $100 \mathrm{mM} \mathrm{NaCl}$. For this purpose, first duplex d(CTGACGCA *CTGC) (8) $3^{\prime}$-d(GACTGCGAGACG) (12) (1 nmole) was dissolved in $0.1 \mathrm{M}$ phosphate buffer $(5 \mu \mathrm{L})$ and $1 \mathrm{M} \mathrm{NaCl}(5 \mu \mathrm{L})$ and the total volume adjusted to $50 \mu \mathrm{L}$ with millipore water. The solution was treated with a freshly prepared solution of NBS or $\mathrm{N}$-iodosuccinimide (NIS) in millipore water and crosslink formation was monitored by RP-HPLC (Fig. 2).

Upon addition of two equivalents of NBS, partial conversion of the non-crosslinked duplex (panel C) into a new species was noticed after 30 minutes (panel D). The expected crosslinked character of the major species was confirmed by gel electrophoresis and mass spectrometric analysis (vide infra and data in ESI $\dagger$ ). Despite the promising outcome of the crosslink experiment, we anticipated that NIS could give faster ICL formation and consequently the effectivity of NBS and NIS was compared for duplex (8).(12). It was observed that on average up to six equivalents of NBS are required for complete

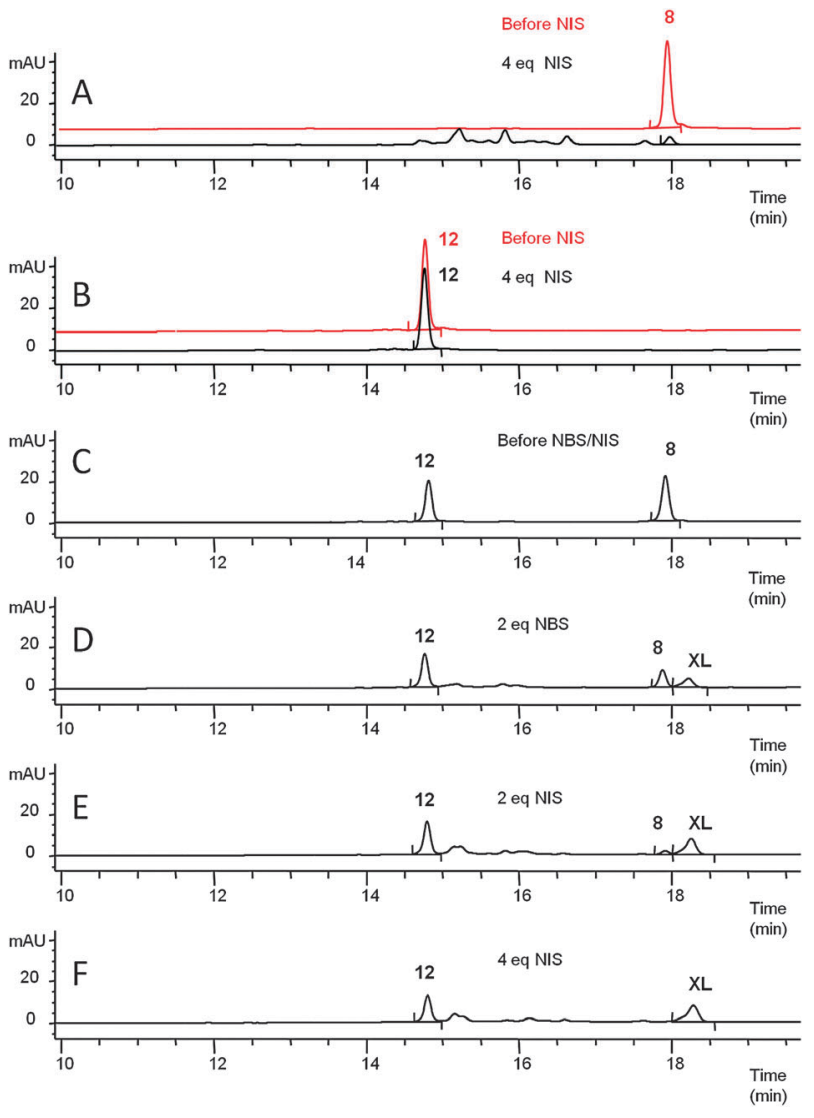

Fig. 2 RP-HPLC analysis of crosslink reactions with NBS and NIS: (A and B) 8 and $\mathbf{1 2}$ before and after NIS addition respectively; (C) $8: 12$ before NBS/NIS addition, $t=t_{0}$; (D and E) $8: 12$ after 2 equiv. of NBS and NIS respectively, $t=t_{0}+30 \mathrm{~min}$; (F) $8: 12$ after 4 equiv. of NIS, $t=t_{0}+60 \mathrm{~min}$. All ODN sequences are mixed in a $1: 1$ ratio to obtain a $20 \mu \mathrm{M}$ final duplex concentration.

consumption of the furan-modified strand (ESI $\dagger$ ). In contrast, generally four equivalents of NIS sufficed for complete conversion, thereby clearly outperforming NBS.

Therefore, NIS became the oxidant of choice to complete a series of crosslinking reactions evaluating (8) to (11) against complements with a varying opposite base. It was observed that in all duplexes featuring $\mathrm{A}$ or $\mathrm{C}$ as an opposite partner, a crosslink peak appeared. In contrast, opposite to $T$ or $G$, no crosslink was formed and modified ONs were completely degraded to a mixture of products (ESI $\dagger$ ). As crosslinked products in all cases show similar retention times as the original furan-modified strands, the degradation of a non-crosslinked furan-modified strand renders purification of the crosslinked material facile, leading to isolation of pure crosslinked duplexes in yields of $15-25 \%$. In all cases, the crosslink peaks for $\mathrm{A}^{*}$ opposite to A showed higher intensity on HPLC than opposite to C. Crosslinking experiments were also analyzed by denaturing PAGE. Fig. 3 shows slower-migrating crosslinked species (as confirmed by ESI-MS analysis) when A and C are opposite partners in a duplex with oligonucleotide $\mathbf{8}$.

As described earlier for cis-2-butene-1,4-dial, crosslinked adducts are formed after initial attack of an exocyclic amine on a cis-2-buten-4-oxo-1-al (Fig. 3, right), followed by Michael addition, resulting in bi- (in case of C) or tricyclic (in case of A) 


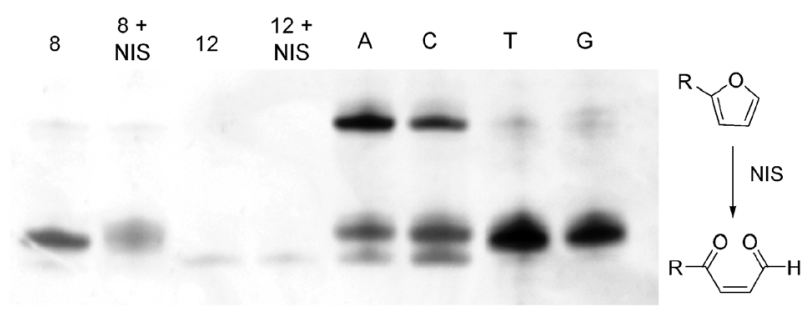

Fig. 3 ICL-formation of $\mathbf{8}$, analyzed by $20 \%$ denaturing PAGE, with A* opposite to A, C, T and G in a duplex DNA (lanes 5-8). Far right: structure of reactive ketoenal formed upon oxidation of a furan moiety.

structures. ${ }^{17,21}$ Considering duplex stability, internal incorporation of the $2^{\prime}-O$-(3-propyl)-furan modified nucleoside leads to more stable duplexes than internal incorporation of the furan-modified $2^{\prime}$-amido-uridine in similar sequences $\left(\Delta T_{\mathrm{m}}\right.$ varying between -3.6 and $\left.-19.9{ }^{\circ} \mathrm{C}\right) .{ }^{22}$ The most stable duplexes are formed when $\mathrm{A}^{*}$ is incorporated in between two guanosines (11) and placed opposite to A or C. This increase in stability does not affect $\mathrm{A}$ or $\mathrm{C}$ crosslinking selectivity, therefore excluding the possibility of correlation between duplex stability and selectivity in ICL formation.

In conclusion, we have developed a straightforward synthesis of a $2^{\prime}$-O-furanylpropyl adenosine building block. Incorporation into double-stranded DNA reduced duplex stability loss in comparison with the previously reported $2^{\prime}$-amido-uridine and acyclic furan-modified building blocks. Incorporation within a $\mathrm{G}\left(\mathbf{A}^{*}\right) \mathrm{G} / \mathrm{CCC}$ context even leads to a $>5{ }^{\circ} \mathrm{C}$ stabilisation of the 12-mer duplex. The current investigation further identified NIS as a superior oxidant over NBS in terms of stoichiometry for the crosslinking reaction.

The selectivity of this new furan-modified purine derivative in furan-oxidation crosslinking was evaluated to show preferential reaction with $\mathrm{A}$ and $\mathrm{C}$ (with a surprising slight preference for crosslinking to A). Though in this specific case, yields are not the highest, a combination of features renders the furanoxidation crosslink methodology very attractive. Benefitting from an induced reactivity principle and proximity being a prerequisite for crosslinking, we have demonstrated that only equimolar amounts of modified strands are necessary to produce substantial amounts of crosslinked material without collateral damage or undesired side reactions. Formation of stable crosslinked duplexes is fast and purification is facile in view of the 'self-destructing' behaviour of the furan-modified strands. As illustrated by current and previous work, ${ }^{16,17,22}$ construction of the furan modified nucleoside building blocks benefits from the commercial availability of a broad range of furan derivatives and follows a very modular and straightforward synthetic route. There is no need for orthogonal protective group manipulations as the furan moiety is completely stable to conditions for phosphoramidite and oligonucleotide synthesis. Fine-tuning of duplex stability, crosslink selectivity and yield is thus possible depending on the specific choice of the building block and desired target.

From current and previous studies on furan modification, it appears plausible to use a $2^{\prime}$-amido-linker for pyrimidine nucleoside and a $2^{\prime}$-O-alkyl-linker for purine nucleoside modification. As such, in view of its synthetic accessibility, its complementarity with the earlier developed furan-modified uridine, its more beneficial influence on duplex stability and its observed crosslink selectivity, the here described $2^{\prime}$ - $O$-furanylpropyladenosine is a valuable addition to our recently developed toolbox for furan-oxidation induced crosslinking.

\section{Notes and references}

1 S. Verma and F. Eckstein, Annu. Rev. Biochem., 1998, 67, 99.

2 C. J. Wilds, A. M. Noronha, S. Robidoux and P. S. Miller, J. Am. Chem. Soc., 2004, 126, 9257.

3 A. M. Noronha, D. M. Noll and P. S. Miller, Nucleosides, Nucleotides Nucleic Acids, 2001, 20, 1303.

4 X. C. Weng, L. G. Ren, L. W. Weng, J. Huang, S. G. Zhu, X. Zhou and L. H. Weng, Angew. Chem., Int. Ed., 2007, 46, 8020.

5 Z. B. Song, X. C. Weng, L. W. Weng, J. Huang, X. L. Wang, M. H. Bai, Y. Y. Zhou, G. F. Yang and X. Zhou, Chem.-Eur. J., 2008, 14, 5751.

6 E. J. Sontheimer and J. A. Steitz, Science, 1993, 262, 1989.

7 M. C. Willis, B. J. Hicke, O. C. Uhlenbeck, T. R. Cech and T. H. Koch, Science, 1993, 262, 1255.

8 P. V. Sergiev, I. N. Lavrik, V. A. Wlassov, S. S. Dokudovskaya, O. A. Dontsova, A. A. Bogadonov and R. Brimacombe, $R N A$, 1997, 3, 464.

9 J. Wower, S. S. Hixson and R. A. Zimmermann, Proc. Natl. Acad. Sci. U. S. A., 1989, 86, 5232.

10 S. E. Osborne, J. Volker, S. Y. Stevens, K. J. Breslauer and G. D. Glick, J. Am. Chem. Soc., 1996, 118, 11993.

11 S. Alefelder and S. T. Sigurdsson, Bioorg. Med. Chem., 2000, 8, 269.

12 X. Peng, I. S. Hong, H. Li, M. M. Seidman and M. M. Greenberg, J. Am. Chem. Soc., 2008, 130, 10299.

13 L. Guan and M. M. Greenberg, J. Am. Chem. Soc., 2009, 131, 15225.

14 Y. Taniguchi, Y. Kurose, T. Nishioka, F. Nagatsugi and S. Sasaki, Bioorg. Med. Chem., 2010, 18, 2894.

15 K. Hattori, T. Hirohama, S. Imoto, S. Kusano and F. Nagatsugi, Chem. Commun., 2009, 6463.

16 S. Halila, T. Velasco, P. D. Clercq and A. Madder, Chem. Commun., 2005, 936.

17 K. Stevens and A. Madder, Nucleic Acids Res., 2009, 37, 1555.

18 A. M. Jawalekar, N. Meeuwenoord, J. S. G. O. Cremers, H. S. Overkleeft, G. A. van der Marel, F. P. J. T. Rutjes and F. L. van Delft, J. Org. Chem., 2008, 73, 287.

19 K. Fuji, T. Morimoto, K. Tsutsumi and K. Kakiuchi, Chem. Commun., 2005, 3295.

20 S. Ke and R. M. Wartell, Nucleic Acids Res., 1993, 21, 5137.

21 M. C. Byrns, D. P. Predecki and L. A. Peterson, Chem. Res. Toxicol., 2002, 15, 373.

22 M. Op de Beeck and A. Madder, J. Am. Chem. Soc., 2011, 133, 796. 